\title{
Some classes of multilinear operators on $C(K)$ spaces
}

\author{
by \\ Fernando Bombal (Madrid), Maite Fernández (Guanajuato), \\ and Ignacio Villanueva (Madrid)
}

\begin{abstract}
We obtain a classification of projective tensor products of $C(K)$ spaces according to whether none, exactly one or more than one factor contains copies of $\ell_{1}$, in terms of the behaviour of certain classes of multilinear operators on the product of the spaces or the verification of certain Banach space properties of the corresponding tensor product. The main tool is an improvement of some results of Emmanuele and Hensgen on the reciprocal Dunford-Pettis and Pełczyński's (V) properties of the projective tensor product of Banach spaces. We also study relationships between several classes of multilinear operators and the associated linear operators.
\end{abstract}

1. Introduction. In recent years much research has been done in the theory of multilinear operators and polynomials between Banach spaces. In particular, various classes of multilinear operators or polynomials have been defined which extended the corresponding notions for linear operators, and the relations between some of these classes have been studied. If $E, F$ and $X$ are Banach spaces and $T: E \times F \rightarrow X$ is a bilinear operator, it is well known that there exists a unique linear operator $\widehat{T}: E \hat{\otimes}_{\pi} F \rightarrow X$ canonically associated to $T$, where $E \hat{\otimes}_{\pi} F$ is the projective tensor product of $E$ and $F$. In Section 2 we improve some results of Emmanuele and Hensgen to establish, under suitable conditions, some non-trivial relationships between several classes of bilinear operators. In Section 3 we use the results of Section 2 to obtain a classification of projective tensor products of several $C(K)$ spaces, according to whether none, exactly one or more than one factor contains copies of $\ell_{1}$, in terms of the behaviour of certain classes of multilinear operators on the product of the spaces or the verification of certain Banach space properties of the corresponding tensor product. Finally, in Section 4 we study the relation between $\widehat{T}$ belonging to certain operator ideals and $T$ belonging to certain classes of multilinear operators.

2000 Mathematics Subject Classification: 46B25, 46B28.

Key words and phrases: tensor products, $C(K)$ spaces.

The first and third authors are partially supported by DGICYT grant PB97-0240. The second author is partially supported by Conacyt Grant J32150-E. 
The notations and terminology used along the paper are standard in Banach space theory, as for instance in [9]. However, before going any further, we recall some notions and notations. $\mathcal{L}^{k}\left(E_{1} \ldots, E_{k} ; X\right)$ will be the Banach space of all continuous $k$-linear mappings from $E_{1} \times \ldots \times E_{k}$ into $X$, and $\mathcal{L}_{\mathrm{wc}}^{k}\left(E_{1}, \ldots, E_{k} ; X\right)$ the closed subspace formed by the weakly compact multilinear operators. When $X=\mathbb{K}$ or $k=1$, we omit them in notation. We write $\mathcal{K}(E ; X)$ for the space of compact operators from $E$ into $X$. As usual, $E_{1} \hat{\otimes}_{\pi} \ldots \hat{\otimes}_{\pi} E_{k}$ stands for the (complete) projective tensor product of the Banach spaces $E_{1}, \ldots, E_{k}$. If $T \in \mathcal{L}^{k}\left(E_{1}, \ldots, E_{k} ; X\right)$ we denote by $\widehat{T}: E_{1} \hat{\otimes}_{\pi} \ldots \hat{\otimes}_{\pi} E_{k} \rightarrow X$ its linearization.

We say that $T \in \mathcal{L}^{k}\left(E_{1}, \ldots, E_{k} ; X\right)$ is completely continuous, and we write $T \in \mathcal{L}_{\mathrm{cc}}^{k}\left(E_{1}, \ldots, E_{k} ; X\right)$, if, given weak Cauchy sequences $\left(x_{i}^{n}\right)_{n \in \mathbb{N}} \subset E_{i}$ $(1 \leq i \leq k)$, the sequence $\left(T\left(x_{1}^{n}, \ldots, x_{k}^{n}\right)\right)_{n}$ is norm convergent in $X$. This definition may be adapted to polynomials in an obvious way. The space of completely continuous polynomials is denoted by $\mathcal{P}_{\text {cc }}\left({ }^{k} E ; X\right)$. By the polarization formula [17, Theorem 1.10], a polynomial is completely continuous if and only if so is its associated symmetric multilinear operator. If $X=\mathbb{K}$, i.e., if $T$ is a multilinear form, we will use the term weakly sequentially continuous instead of completely continuous.

If $T \in \mathcal{L}^{k}\left(E_{1}, \ldots, E_{k} ; X\right)$ we denote by $T_{i}(1 \leq i \leq k)$ the operator $T_{i} \in \mathcal{L}\left(E_{i} ; \mathcal{L}^{k-1}\left(E_{1}, .{ }^{[i]} ., E_{k} ; X\right)\right)$ defined by

$$
T_{i}\left(x_{i}\right)\left(x_{1},{ }^{[i]} ., x_{k}\right):=T\left(x_{1}, \ldots, x_{k}\right) .
$$

We shall say that $T$ is regular if all the maps $T_{i}, 1 \leq i \leq k$, defined above are weakly compact.

Recall that $E$ has the Dunford-Pettis property (DPP for short) if, for every $X, \mathcal{L}_{\mathrm{wc}}(E ; X) \subseteq \mathcal{L}_{\mathrm{cc}}(E ; X)$. Examples of spaces with the DPP are $C(K)$ and $L_{1}(\mu)$ spaces. $E$ has the reciprocal Dunford-Pettis property $(R D P P$ for short) if, for every $X, \mathcal{L}_{\mathrm{cc}}(E ; X) \subseteq \mathcal{L}_{\mathrm{wc}}(E ; X)$. The spaces containing no copy of $\ell_{1}$, and $C(K)$ spaces have the RDPP. Both properties were introduced in [14].

A formal series $\sum x^{n}$ in a Banach space $E$ is weakly unconditionally Cauchy (w.u.C. for short) if there is $C>0$ such that, for any finite subset $\Delta$ of $\mathbb{N}$ and any signs \pm , we have $\left\|\sum_{n \in \Delta} \pm x^{n}\right\| \leq C$. For other equivalent definitions, see [8, Theorem V.6]. The series $\sum x^{n}$ is unconditionally convergent if every subseries is norm convergent. Other equivalent definitions may be found in [9, Theorem 1.9].

A linear operator between Banach spaces is unconditionally converging if it takes w.u.C. series to unconditionally convergent series. A Banach space $E$ is said to have Pełczyński's property (V) if every unconditionally converging linear operator on $E$ is weakly compact. This property was introduced in 
[18], where it is shown that $C(K)$ spaces have property $(\mathrm{V})$, and that the dual of a space with property $(\mathrm{V})$ is weakly sequentially complete.

Following [12], we say that $T \in \mathcal{L}^{k}\left(E_{1}, \ldots, E_{k} ; X\right)$ is unconditionally converging if, given w.u.C. series $\sum_{n \in \mathbb{N}} x_{i}^{n}$ in $E_{i}(1 \leq i \leq k)$, the sequence

$$
\left(T\left(s_{1}^{m}, \ldots, s_{k}^{m}\right)\right)_{m}
$$

is norm convergent in $X$, where $s_{i}^{m}=\sum_{n=1}^{m} x_{i}^{n}$. This definition may be adapted to polynomials in an obvious way. Since a linear operator fails to be unconditionally converging if and only if it preserves a copy of $c_{0}[8$, Exercise V.8], it is clear that the definition of unconditionally converging $k$-linear operators agrees for $k=1$ with that of unconditionally converging linear operators.

By the polarization formula, a polynomial is unconditionally converging if and only if so is its associated symmetric multilinear operator.

Since $B_{E \hat{\otimes}_{\pi} F}=\overline{\operatorname{coe}}\left(B_{E} \otimes B_{F}\right)$, it follows that $T$ is (weakly) compact if and only if $\widehat{T}$ is (weakly) compact.

2. Some properties of bilinear operators. In [10] and [11], the following results are proved:

THEOREM 2.1. Let $E$ be a Banach space not containing $\ell_{1}$ and $F$ a $B a$ nach space with the RDPP. If $\mathcal{L}\left(E ; F^{*}\right)=\mathcal{K}\left(E ; F^{*}\right)$, then $E \hat{\otimes}_{\pi} F$ has the $R D P P$.

Theorem 2.2. Let $E, F$ be Banach spaces with the RDPP such that $E^{*}$ and $F^{*}$ are weakly sequentially complete. If $\mathcal{L}\left(E ; F^{*}\right)=\mathcal{K}\left(E ; F^{*}\right)$, then $E \hat{\otimes}_{\pi} F$ has the RDPP.

Theorem 2.3. Let E, F be Banach spaces with property $(\mathrm{V})$ such that $\mathcal{L}\left(E ; F^{*}\right)=\mathcal{K}\left(E ; F^{*}\right)$. Then $E \hat{\otimes}_{\pi} F$ has property $(\mathrm{V})$.

Taking advantage of the ideas in those papers, we now prove a strengthening of these results. First we will need some definitions and lemmas.

Definition 2.4. Let $E$ be a Banach space. A set $M \subset E^{*}$ is an $L$-set (respectively a $V$-set) if for every weakly null sequence $\left(x_{n}\right) \subset E$ (resp. every w.u.C. series $\sum_{n} x_{n} \subset E$ ), we have

$$
\lim _{n \rightarrow \infty} \sup \left\{\left|x^{*}\left(x_{n}\right)\right|: x^{*} \in M\right\}=0 .
$$

The following result is well known.

Proposition 2.5. Let $E$ be a Banach space. Then:

(a) $E$ has the RDPP if and only if every L-set in $E^{*}$ is relatively weakly compact ([16], see also [3]).

(b) E has property (V) if and only if every $V$-set in $E^{*}$ is relatively weakly compact ([18]). 
We will also need some results concerning the Aron-Berner extension of a multilinear operator: if $T: E \times F \rightarrow X$ is a bilinear operator, then we can define its Aron-Berner extension

$$
\mathrm{AB}(T): E^{* *} \times F^{* *} \rightarrow X^{* *}
$$

by

$$
\mathrm{AB}(T)\left(z_{1}, z_{2}\right)=\lim _{\alpha} \lim _{\beta} T\left(x_{\alpha}, y_{\beta}\right),
$$

where $\left(x_{\alpha}\right) \subset E$ is a net weak-star converging to $z_{1}$ and $\left(y_{\beta}\right) \subset F$ is a net weak-star converging to $z_{2}$. We will use the following results from [15] relating to this extension.

Lemma 2.6. Let $E, F$ be Banach spaces with the $R D P P$, and $X$ any $B a$ nach space. If $T: E \times F \rightarrow X$ is a completely continuous bilinear operator, then its Aron-Berner extension $\mathrm{AB}(T)$ takes values in $X$.

Lemma 2.7. Let $E, F$ be Banach spaces whose duals $E^{*}$ and $F^{*}$ have the Dunford-Pettis property and $\mathcal{L}\left(E ; F^{*}\right)=\mathcal{L}_{\text {wc }}\left(E ; F^{*}\right)$. For any Banach space $X$, if $T: E \times F \rightarrow X$ is a bilinear operator such that $\mathrm{AB}(T)$ is $X$-valued, then $\mathrm{AB}(T): E^{* *} \times F^{* *} \rightarrow X$ is completely continuous.

Lemma 2.8. Let $E, F$ be Banach spaces with property (V), and $X$ any Banach space. If $T: E \times F \rightarrow X$ is an unconditionally converging bilinear operator, then $\mathrm{AB}(T)$ takes values in $X$.

Lemma 2.9. Let $E, F$ be Banach spaces such that $\mathcal{L}\left(E ; F^{*}\right)=$ $\mathcal{L}_{\mathrm{wc}}\left(E ; F^{*}\right)$. For any Banach space $X$, if $T: E \times F \rightarrow X$ is a bilinear operator such that $\mathrm{AB}(T)$ is $X$-valued, then $\mathrm{AB}(T): E^{* *} \times F^{* *} \rightarrow X$ is unconditionally converging.

Now we can prove the following.

Proposition 2.10. Let $E$ be a Banach space not containing $\ell_{1}$ and $F$ a Banach space with the RDPP. Assume further that $\mathcal{L}\left(E ; F^{*}\right)=\mathcal{K}\left(E ; F^{*}\right)$ and that $E^{*}$ and $F^{*}$ have the Dunford-Pettis property. For every Banach space $X$, if $T: E \times F \rightarrow X$ is a completely continuous bilinear operator, then $T$ is weakly compact.

Proof. Let $T$ be as in the hypothesis and let $\widehat{T}: E \hat{\otimes}_{\pi} F \rightarrow X$ be the operator canonically associated to $T$. Since $T$ is weakly compact if and only if $\widehat{T}$ is, it suffices to prove that $\widehat{T}^{*}$ is weakly compact. Let then $M=\widehat{T}^{*}\left(B_{X^{*}}\right) \subset$ $\left(E \hat{\otimes}_{\pi} F\right)^{*}=\mathcal{K}\left(E ; F^{*}\right)$. Let $\left(h_{n}\right)_{n} \subset M$ and let $\left(\varphi_{n}\right)_{n} \subset B_{X^{*}}$ be such that $\widehat{T}^{*}\left(\varphi_{n}\right)=h_{n}$ for every $n \in \mathbb{N}$. Define $H=\overline{\operatorname{span}}\left[h_{n}(x): x \in E, n \in \mathbb{N}\right]$. Then $H$ is a closed subspace of $F^{*}$ and $H$ is separable, because, for every $n \in \mathbb{N}, h_{n}: E \rightarrow F^{*}$ is compact. Let now $Y \subset F$ be a countable norming set of $H$ and let $y \in Y$.

Claim 1. The set $\left\{h_{n}^{*}(y) ; n \in \mathbb{N}\right\} \subset E^{*}$ is an L-set. 
Proof. Let $\left(x_{m}\right)_{m} \subset E$ be a sequence weakly converging to 0 . We have

$$
\begin{aligned}
h_{n}^{*}(y)\left(x_{m}\right) & =h_{n}\left(x_{m}\right)(y)=\widehat{T}^{*}\left(\varphi_{n}\right)\left(x_{m} \otimes y\right)=\left\langle\widehat{T}\left(x_{m} \otimes y\right), \varphi_{n}\right\rangle \\
& =\left\langle T\left(x_{m}, y\right), \varphi_{n}\right\rangle .
\end{aligned}
$$

Therefore

$$
\lim _{m \rightarrow \infty} \sup _{n \in \mathbb{N}}\left|h_{n}^{*}(y)\left(x_{m}\right)\right| \leq \lim _{m \rightarrow \infty}\left\|T\left(x_{m}, y\right)\right\|=0,
$$

and the claim is proved.

So $\left\{h_{n}^{*}(y) ; n \in \mathbb{N}\right\} \subset E^{*}$ is relatively weakly compact and therefore we can suppose (using the fact that $Y$ is countable and considering subsequences if necessary) that, for every $y \in Y,\left(h_{n}^{*}(y)\right)_{n}$ is a weakly Cauchy sequence. Let now $x^{* *} \in E^{* *}$.

Claim 2. The set $\left\{h_{n}^{* *}\left(x^{* *}\right) ; n \in \mathbb{N}\right\} \subset F^{*}$ is an L-set.

Proof. If we think of $h_{n} \in \mathcal{K}\left(E ; F^{*}\right)$ as a bilinear form, $h_{n}: E \times F \rightarrow \mathbb{K}$, it is clear that $h_{n}^{* *}\left(x^{* *}\right)(y)=\mathrm{AB}\left(h_{n}\right)\left(x^{* *}, y\right)$, where $\mathrm{AB}\left(h_{n}\right)$ denotes any of the two Aron-Berner extensions of $h_{n}$. Let then $\left(y_{m}\right)_{m} \subset F$ be a sequence weakly converging to 0 . Then

$$
h_{n}^{* *}\left(x^{* *}\right)\left(y_{m}\right)=\operatorname{AB}\left(\widehat{T}^{*}\left(\varphi_{n}\right)\right)\left(x^{* *}, y_{m}\right) .
$$

Let us see now that $\operatorname{AB}\left(\widehat{T}^{*}\left(\varphi_{n}\right)\right)\left(x^{* *}, y_{m}\right)=\left\langle\operatorname{AB}(T)\left(x^{* *}, y_{m}\right), \varphi_{n}\right\rangle$ : let $\left(x_{\alpha}\right)_{\alpha}$ $\subset E$ be a bounded net weak-star convergent to $x^{* *}$. Then

$$
\begin{aligned}
\operatorname{AB}\left(\widehat{T}^{*}\left(\varphi_{n}\right)\right)\left(x^{* *}, y_{m}\right) & =\lim _{\alpha} \widehat{T}^{*}\left(\varphi_{n}\right)\left(x_{\alpha}, y_{m}\right)=\lim _{\alpha}\left\langle\widehat{T}\left(x_{\alpha} \otimes y_{m}\right), \varphi_{n}\right\rangle \\
& =\lim _{\alpha}\left\langle T\left(x_{\alpha}, y_{m}\right), \varphi_{n}\right\rangle=\left\langle\operatorname{AB}(T)\left(x^{* *}, y_{m}\right), \varphi_{n}\right\rangle .
\end{aligned}
$$

Therefore,

$$
\lim _{m \rightarrow \infty} \sup _{n \in \mathbb{N}}\left|h_{n}^{* *}\left(x^{* *}\right)\left(y_{m}\right)\right| \leq \lim _{m \rightarrow \infty}\left\|\operatorname{AB}(T)\left(x^{* *}, y_{m}\right)\right\|=0 .
$$

The last limit is 0 because $\mathrm{AB}(T)$ is completely continuous, as follows from Lemmas 2.6 and 2.7. So, the claim is proved.

Now we can proceed as in [10] to obtain an $h \in \mathcal{K}\left(E ; F^{*}\right)$ such that $h_{n}$ weakly converges to $h$, which finishes the proof.

Corollary 2.11. Let E, F be Banach spaces with the RDPP such that $E^{*}$ and $F^{*}$ are weakly sequentially complete and have the Dunford-Pettis property. Assume further that $\mathcal{L}\left(E ; F^{*}\right)=\mathcal{K}\left(E ; F^{*}\right)$. For every Banach space $X$, if $T: E \times F \rightarrow X$ is a completely continuous bilinear operator, then $T$ is weakly compact.

Proof. The beginning of the proof runs as the proof that $\left(h_{n}\right)_{n}$ is weakly Cauchy in Proposition 2.10. To finish it, we reason as in the proof of Theorem 2.2 (see [10, Cor. 4]). 
Since compact operators are completely continuous, Proposition 4.1 below implies that our results are indeed a strengthening (under the additional hypothesis that $E^{*}$ and $F^{*}$ have the DP property) of Theorems 2.1 and 2.2. For instance, note that, since $c_{0} \hat{\otimes}_{\pi} \ell_{\infty}$ does not have the DP property $([6])$, there are completely continuous bilinear operators defined on $c_{0} \times \ell_{\infty}$ such that the associated linear operators defined on $c_{0} \hat{\otimes}_{\pi} \ell_{\infty}$ are not completely continuous.

We have a similar result for unconditionally converging bilinear operators. This time we do not need additional hypotheses on $E$ and $F$.

Proposition 2.12. Let E, F be Banach spaces with property (V). Assume further that $\mathcal{L}\left(E ; F^{*}\right)=\mathcal{K}\left(E ; F^{*}\right)$. For every Banach space $X$, if $T: E \times F \rightarrow X$ is an unconditionally converging bilinear operator, then $T$ is weakly compact.

Proof. As in the proof of Proposition 2.10, it suffices to prove that $\widehat{T}^{*}\left(B_{X^{*}}\right)=M \subset\left(E \hat{\otimes}_{\pi} F\right)^{*}=\mathcal{K}\left(E ; F^{*}\right)$ is relatively weakly compact. Let then $h_{n}, \varphi_{n}, H$ and $Y$ be as in the proof of Proposition 2.10.

Claim 1. The set $\left\{h_{n}^{*}(y) ; n \in \mathbb{N}\right\} \subset E^{*}$ is a $V$-set.

Proof. Let $\sum_{m} x_{m} \subset E$ be a w.u.C. series. As in the proof of Proposition 2.10,

$$
h_{n}^{*}(y)\left(x_{m}\right)=h_{n}\left(x_{m}\right)(y)=\left\langle T\left(x_{m}, y\right), \varphi_{n}\right\rangle .
$$

It is very easy to see that $T$ is separately unconditionally converging, so

$$
\lim _{m \rightarrow \infty} \sup _{n \in \mathbb{N}}\left|h_{n}^{*}(y)\left(x_{m}\right)\right| \leq \lim _{m \rightarrow \infty}\left\|T\left(x_{m}, y\right)\right\|=0,
$$

and the claim is proved.

So $\left\{h_{n}^{*}(y) ; n \in \mathbb{N}\right\} \subset E^{*}$ is relatively weakly compact and again as in the proof of Proposition 2.10 we can suppose that, for every $y \in Y$, $\left(h_{n}^{*}(y)\right)_{n} \subset E^{*}$ is a weakly Cauchy sequence. Let now $x^{* *} \in E^{* *}$.

Claim 2. The set $\left\{h_{n}^{* *}\left(x^{* *}\right) ; n \in \mathbb{N}\right\} \subset F^{*}$ is a $V$-set.

Proof. Observe that it follows from Lemmas 2.8 and 2.9 that $\operatorname{AB}(T)$ is unconditionally converging, hence separately unconditionally converging. So, proceeding as in the proof of Proposition 2.10 we get

$$
\lim _{m \rightarrow \infty} \sup _{n \in \mathbb{N}}\left|h_{n}^{* *}\left(x^{* *}\right)\left(y_{m}\right)\right| \leq \lim _{m \rightarrow \infty}\left\|\operatorname{AB}(T)\left(x^{* *}, y_{m}\right)\right\|=0,
$$

and the claim is proved.

Now we can proceed as in [11] to obtain $h \in \mathcal{K}\left(E ; F^{*}\right)$ such that $h_{n}$ weakly converges to $h$, which finishes the proof.

From Theorem 4.2 below it follows that Proposition 2.12 is strictly stronger than Theorem 2.3. 
3. Projective tensor products of $C(K)$ spaces. This section was the original motivation of this paper. We now apply the results of the previous sections to obtain a classification of projective tensor products of $C(K)$ spaces in terms of some classical Banach space properties.

It is known that the projective tensor product of Banach spaces is associative, that is, if $E, F, G$ are Banach spaces, then $E \hat{\otimes}_{\pi} F \hat{\otimes}_{\pi} G=E \hat{\otimes}_{\pi}$ $\left(F \hat{\otimes}_{\pi} G\right)=\left(E \hat{\otimes}_{\pi} F\right) \hat{\otimes}_{\pi} G$. We will make frequent use of this fact.

Recall that a compact Hausdorff space $K$ is said to be scattered (or dispersed) if it does not contain any non-void perfect set. In [19] it is proved, among other interesting results, that $K$ is scattered if and only if $C(K)$ contains no copy of $\ell_{1}$. In this case, $C(K)^{*}$ can be identified with $\ell_{1}(\Gamma)$ for some $\Gamma$ and, consequently, it is a Schur space.

Theorem 3.1. Let $k \geq 2$ and $K_{1}, \ldots, K_{k}$ be infinite compact Hausdorff spaces. Then the following assertions are equivalent:

$\left(\mathrm{a}_{1}\right)$ For every $i \in\{1, \ldots, k\}, K_{i}$ is scattered.

( $\left.\mathrm{b}_{1}\right) C\left(K_{1}\right) \hat{\otimes}_{\pi} \ldots \hat{\otimes}_{\pi} C\left(K_{k}\right)$ has properties DP, RDP and $(\mathrm{V})$.

$\left(c_{1}\right) C\left(K_{1}\right) \hat{\otimes}_{\pi} \ldots \hat{\otimes}_{\pi} C\left(K_{k}\right)$ does not contain any isomorphic copy of $\ell_{1}$.

$\left(\mathrm{d}_{1}\right)$ For any Banach space $X$, and any k-linear operator $T: C\left(K_{1}\right) \times$ $\ldots \times C\left(K_{k}\right) \rightarrow X$ the following are equivalent:

(1) $T$ is completely continuous.

(2) $T$ is unconditionally converging.

(3) $T$ is weakly compact.

(4) $T$ is regular.

(5) $T$ is compact.

Proof. We will first prove that $\left(\mathrm{a}_{1}\right)$ implies all the others. By a standard argument (see, for instance, the proof of [5, Theorem 3.1]), it can be proved that

$$
\begin{aligned}
\left(C\left(K_{1}\right) \hat{\otimes}_{\pi} \ldots \hat{\otimes}_{\pi} C\left(K_{k}\right)\right)^{*} & =C\left(K_{1}\right)^{*} \check{\otimes}_{\varepsilon} \ldots \check{\otimes}_{\varepsilon} C\left(K_{k}\right)^{*} \\
& =\mathcal{K}\left(C\left(K_{1}\right) ;\left(C\left(K_{2}\right) \hat{\otimes}_{\pi} \ldots \hat{\otimes}_{\pi} C\left(K_{k}\right)\right)^{*}\right) .
\end{aligned}
$$

Hence, $\left(C\left(K_{1}\right) \hat{\otimes}_{\pi} \ldots \hat{\otimes}_{\pi} C\left(K_{k}\right)\right)^{*}$ is a Schur space, and so, by a well known result of Pethe and Thakare, $X:=C\left(K_{1}\right) \hat{\otimes}_{\pi} \ldots \hat{\otimes}_{\pi} C\left(K_{k}\right)$ has the DPP and contains no copy of $\ell_{1}$. Also, from the associativity of projective tensor products and Theorem 2.3 it follows that $X$ has property $(\mathrm{V})$, and hence it also has the RDPP. So $\left(b_{1}\right)$ and $\left(c_{1}\right)$ hold.

Let us check $\left(\mathrm{d}_{1}\right)$ : Compact multilinear operators are always weakly compact, and weakly compact multilinear operators are completely continuous on a product of spaces with the DP property. On $C(K)$ spaces, multilinear unconditionally converging and completely continuous operators coincide ([15]). Since no $C\left(K_{i}\right)(1 \leq i \leq k)$ contains copies of $\ell_{1}$ by hypothesis, every completely continuous multilinear operator on $C\left(K_{1}\right) \times \ldots \times C\left(K_{k}\right)$ is 
compact. On $C(K)$ spaces, every regular multilinear operator is completely continuous ([5, Lemma 2.6]). Finally, reasoning as in [2] we can prove that, under the assumption $\left(\mathrm{a}_{1}\right)$, if $T$ is completely continuous, it is weakly continuous on bounded sets ([2, Proposition 2.12]), hence regular ([2, Theorem 2.9]).

For the converse implications, notice that one and only one of the conditions $\left(\mathrm{a}_{1}\right)$, or $\left(\mathrm{a}_{2}\right),\left(\mathrm{a}_{3}\right)$ (in Theorems 3.3 and 3.4 below) holds. Then, by exclusion, it is enough to prove that conditions $\left(\mathrm{a}_{i}\right)(i=1,2,3)$ imply all the others in Theorems 3.1, 3.3 and 3.4.

Thanks are due to Joaquín Gutiérrez for his help in shortening the proof of $\left(\mathrm{a}_{1}\right) \Rightarrow\left(\mathrm{d}_{1}\right)$.

Corollary 3.2. Let $K$ be an infinite compact Hausdorff space and $2 \leq$ $k \in \mathbb{N}$. Then the following assertions are equivalent:

(a) $K$ is scattered.

(b) $\hat{\otimes}_{\pi, s}^{k} C(K)$ has properties $D P, R D P$ and $(\mathrm{V})$.

(c) $\hat{\otimes}_{\pi, s}^{k} C(K)$ does not contain any isomorphic copy of $\ell_{1}$.

(d) Any k-homogeneous unconditionally converging polynomial on $C(K)$ is weakly compact.

Proof. Reasoning as in the last part of the proof of Theorem 3.1, it suffices to prove that (the same situation will occur in Corollary 3.5) condition (a) implies all the others. Hence, suppose (a) holds. Since $\hat{\otimes}_{\pi, s}^{k} C(K)$ is complemented in $\hat{\otimes}_{\pi}^{k} C(K)$, (b) and (c) follow. If a polynomial $P: C(K) \rightarrow X$ is unconditionally converging, then its associated symmetric multilinear form $T$ is also unconditionally converging. By Theorem 3.1, $T$ is weakly compact, hence $P$ is weakly compact.

TheOREm 3.3. Let $K_{1}, \ldots, K_{k}$ be compact Hausdorff spaces. Then the following assertions are equivalent:

$\left(\mathrm{a}_{2}\right)$ There exists precisely one $i \in\{1, \ldots, k\}$ such that $K_{i}$ is not scattered (i.e., $\left.C\left(K_{i}\right) \supset \ell_{1}\right)$.

$\left(\mathrm{b}_{2}\right) C\left(K_{1}\right) \hat{\otimes}_{\pi} \ldots \hat{\otimes}_{\pi} C\left(K_{k}\right)$ has properties $R D P$ and $(\mathrm{V})$, but it does not have the DP property.

$\left(c_{2}\right) C\left(K_{1}\right) \hat{\otimes}_{\pi} \ldots \hat{\otimes}_{\pi} C\left(K_{k}\right)$ contains $\ell_{1}$, but not complemented.

$\left(\mathrm{d}_{2}\right)$ For any Banach space $X$ and any k-linear operator $T: C\left(K_{1}\right) \times$ $\ldots \times C\left(K_{k}\right) \rightarrow X, T$ is unconditionally converging (equivalently completely continuous) if and only if $T$ is weakly compact, but there are weakly compact multilinear operators on $C\left(K_{1}\right) \times \ldots \times C\left(K_{k}\right)$ which are neither compact nor regular.

Proof. As mentioned before, we only need to show that $\left(\mathrm{a}_{2}\right)$ implies all the others. Let us prove $\left(b_{2}\right)$ : The statement about the DP property can be 
found in [6]. For properties (V) and RDP, we use induction on $k$. For $k=2$ the result follows from Theorems 2.1 and 2.3. Suppose it is true for $k-1$, and let $C\left(K_{k}\right) \supset \ell_{1}$. From the induction hypothesis it follows that $C\left(K_{2}\right) \hat{\otimes}_{\pi}$ $\ldots \hat{\otimes}_{\pi} C\left(K_{k}\right)$ has property $(\mathrm{V})$, hence it cannot contain complemented copies of $\ell_{1}$. Therefore, $\left(C\left(K_{2}\right) \hat{\otimes}_{\pi} \ldots \hat{\otimes}_{\pi} C\left(K_{k}\right)\right)^{*}$ does not contain copies of $c_{0}$. Then every operator from $C\left(K_{1}\right)$ into $\left(C\left(K_{2}\right) \hat{\otimes}_{\pi} \ldots \hat{\otimes}_{\pi} C\left(K_{k}\right)\right)^{*}$ is compact. Now we use the associativity of the projective tensor product and Theorem 2.3.

Clearly $C\left(K_{1}\right) \hat{\otimes}_{\pi} \ldots \hat{\otimes}_{\pi} C\left(K_{k}\right)$ contains a copy of $\ell_{1}$. But since it has property $(\mathrm{V})$, none of such copies can be complemented. So, $\left(\mathrm{b}_{2}\right)$ implies $\left(\mathrm{c}_{2}\right)$.

Let us now see that $\left(\mathrm{a}_{2}\right)$ implies $\left(\mathrm{d}_{2}\right)$. We first show that, under assumption $\left(\mathrm{a}_{2}\right)$, unconditionally converging multilinear operators on $C\left(K_{1}\right) \times \ldots \times$ $C\left(K_{k}\right)$ are weakly compact. The proof is a refinement of the proof of Proposition 2.12. We apply induction on $k$. For $k=2$ the result has already been proved. Suppose it is true for $k-1$, and let $T: C\left(K_{1}\right) \times \ldots \times C\left(K_{k}\right) \rightarrow X$ be an unconditionally converging multilinear operator and $\widehat{T}$ its linearization. We define

$$
S: C\left(K_{1}\right) \times\left(C\left(K_{2}\right) \hat{\otimes}_{\pi} \ldots \hat{\otimes}_{\pi} C\left(K_{k}\right)\right) \rightarrow X
$$

by

$$
S\left(f_{1}, y\right):=\widehat{T}\left(f_{1} \otimes y\right) .
$$

Clearly, $S$ is bilinear and continuous, with $\|S\|=\|\widehat{T}\|=\|T\|$. Let

$$
\widehat{S}: C\left(K_{1}\right) \hat{\otimes}_{\pi} C\left(K_{2}\right) \hat{\otimes}_{\pi} \ldots \hat{\otimes}_{\pi} C\left(K_{k}\right) \rightarrow X
$$

be the linear operator associated to $S$. Clearly, we just have to check that $\widehat{S}^{*}: X^{*} \rightarrow \mathcal{K}\left(C\left(K_{1}\right) ;\left(C\left(K_{2}\right) \hat{\otimes}_{\pi} \ldots \hat{\otimes}_{\pi} C\left(K_{k}\right)\right)^{*}\right)$ is weakly compact. As before, let $M=\widehat{S}^{*}\left(B_{X^{*}}\right)$, let $\left(\varphi_{n}\right)_{n} \subset B_{X^{*}}$ and let $h_{n}=\widehat{S}^{*}\left(\varphi_{n}\right)$. We just need to extract a weakly converging subsequence from $\left(h_{n}\right)_{n}$. Let $H=$ $\overline{\operatorname{span}}\left[h_{n}\left(f_{1}\right): f_{1} \in C\left(K_{1}\right), n \in \mathbb{N}\right]$. Then $H$ is a separable closed subspace of $\left(C\left(K_{2}\right) \hat{\otimes}_{\pi} \ldots \hat{\otimes}_{\pi} C\left(K_{k}\right)\right)^{*}$. As before, let $Y \subset C\left(K_{2}\right) \hat{\otimes}_{\pi} \ldots \hat{\otimes}_{\pi} C\left(K_{k}\right)$ be a countable norming set of $H$ and let $y \in Y$.

Let us prove that $S_{y}=S(\cdot, y): C\left(K_{1}\right) \rightarrow X$ is unconditionally converging. Since $T$ is separately unconditionally converging, this is clear when $y=f_{2} \otimes \ldots \otimes f_{k}$, and it follows readily for $y=\sum_{i=1}^{n} f_{2}^{i} \otimes \ldots \otimes f_{k}^{i}$. For the general case it suffices to take into account the density of $C\left(K_{2}\right) \otimes \ldots \otimes C\left(K_{k}\right)$ in $C\left(K_{2}\right) \hat{\otimes}_{\pi} \ldots \hat{\otimes}_{\pi} C\left(K_{k}\right)$ and the fact that the canonical continuous linear map

$$
C\left(K_{2}\right) \hat{\otimes}_{\pi} \ldots \hat{\otimes}_{\pi} C\left(K_{k}\right) \ni y \mapsto S_{y} \in \mathcal{L}\left(C\left(K_{1}\right) ; X\right)
$$

takes values in the closed subspace of unconditionally converging operators when $y \in C\left(K_{2}\right) \otimes \ldots \otimes C\left(K_{k}\right)$.

Using this, we can reason as in the proof of Proposition 2.12 to establish that the set $\left\{h_{n}^{*}(y) ; n \in \mathbb{N}\right\} \subset C\left(K_{1}\right)^{*}$ is a $\mathrm{V}$-set. 
So $\left\{h_{n}^{*}(y) ; n \in \mathbb{N}\right\} \subset C\left(K_{1}\right)^{*}$ is relatively weakly compact and we can suppose that, for every $y \in Y,\left(h_{n}^{*}(y)\right)_{n} \subset C\left(K_{1}\right)^{*}$ is a weakly Cauchy sequence. Let now $z \in C\left(K_{1}\right)^{* *}$.

Claim. The set $\left\{h_{n}^{* *}(z) ; n \in \mathbb{N}\right\} \subset\left(C\left(K_{2}\right) \hat{\otimes}_{\pi} \ldots \hat{\otimes}_{\pi} C\left(K_{k}\right)\right)^{*}$ is a V-set.

Proof. Let $\sum_{n} y_{n} \subset C\left(K_{2}\right) \hat{\otimes}_{\pi} \ldots \hat{\otimes}_{\pi} C\left(K_{k}\right)$ be a w.u.C. series. As in the proof of Proposition 2.12, we get

$$
\left|h_{n}^{* *}(z)\left(y_{m}\right)\right| \leq\left\|\mathrm{AB}(\widehat{S})\left(z, y_{m}\right)\right\| .
$$

Since $T$ is unconditionally converging, so is $\mathrm{AB}(T)$ (Lemma 2.9). Therefore $\mathrm{AB}(T)_{z}: C\left(K_{2}\right) \times \ldots \times C\left(K_{k}\right) \rightarrow X$ defined by

$$
\mathrm{AB}(T)_{z}\left(f_{2}, \ldots, f_{k}\right)=\mathrm{AB}(T)\left(z, f_{2}, \ldots, f_{k}\right)
$$

is unconditionally converging. Let $\widehat{\mathrm{AB}(T})_{z}: C\left(K_{2}\right) \hat{\otimes}_{\pi} \ldots \hat{\otimes}_{\pi} C\left(K_{k}\right) \rightarrow X$ be the associated linear operator. By the induction hypothesis, $\widehat{\operatorname{AB}(T)})_{z}$ is weakly compact, hence unconditionally converging. Clearly, for every $y \in$ $C\left(K_{2}\right) \hat{\otimes}_{\pi} \ldots \hat{\otimes}_{\pi} C\left(K_{k}\right)$, we have $\left.\mathrm{AB}(S)(z, y)=\widehat{\mathrm{AB}(T}\right)_{z}(y)$ and so the claim follows.

Now we can again proceed as in [11] to finish the proof that unconditionally converging multilinear operators are weakly compact.

For a weakly compact, neither regular nor compact multilinear operator on $C\left(K_{1}\right) \times \ldots \times C\left(K_{k}\right)$ we proceed similarly to the proof of the main result of [6]: suppose that $C\left(K_{1}\right) \supset \ell_{1}$. Then there exists a surjective operator $q: C\left(K_{1}\right) \rightarrow \ell_{2}\left(\left[9\right.\right.$, Corollary 4.16]). Let $\left(x_{2}^{n}\right) \subset C\left(K_{2}\right)$ and $\left(\mu_{2}^{n}\right) \subset B_{C\left(K_{2}\right)^{*}}$ be two sequences such that $\left(x_{2}^{n}\right)$ converges weakly to 0 and $\mu_{2}^{n}\left(x_{2}^{n}\right)=1$ for every $n \in \mathbb{N}$. Choose norm one elements $\mu_{i} \in C\left(K_{i}\right)^{*}$ and $x_{i}^{0} \in C\left(K_{i}\right)$ such that $\mu_{i}\left(x_{i}^{0}\right)=1(3 \leq i \leq k)$. Then we can consider the multilinear operator

$$
T: C\left(K_{1}\right) \times \ldots \times C\left(K_{k}\right) \rightarrow \ell_{2}
$$

defined by

$$
T\left(x_{1}, \ldots, x_{k}\right)=\left(q\left(x_{1}\right)_{n} \mu_{2}^{n}\left(x_{2}\right) \prod_{i=3}^{k} \mu_{i}\left(x_{i}\right)\right)_{n} .
$$

$T$ is clearly weakly compact. Let $\left(x_{1}^{n}\right) \subset\|q\| B_{C\left(K_{1}\right)}$ be a sequence such that $q\left(x_{1}^{n}\right)=e_{n}$. The sequence $\left(x_{1}^{n} \otimes x_{2}^{n} \otimes x_{3}^{0} \otimes \ldots \otimes x_{k}^{0}\right)_{n} \subset C\left(K_{1}\right) \hat{\otimes}_{\pi} \ldots \hat{\otimes}_{\pi} C\left(K_{k}\right)$ converges weakly to 0 , since $\left(x_{1}^{n} \otimes x_{2}^{n}\right)_{n}$ converges weakly to 0 in $C\left(K_{1}\right) \hat{\otimes}_{\pi}$ $C\left(K_{2}\right)$ ([6, Lemma 2.1]). However,

$$
T\left(x_{1}^{n}, x_{2}^{n}, x_{3}^{0}, \ldots, x_{k}^{0}\right)=1
$$

for every $n$. So, $\widehat{T}$ is not completely continuous. Hence, $\widehat{T}$, and consequently $T$, cannot be compact. 
Moreover, the operator $T_{1}: C\left(K_{2}\right) \rightarrow \mathcal{L}^{k-1}\left(C\left(K_{1}\right), C\left(K_{3}\right), \ldots, C\left(K_{k}\right) ; \ell_{2}\right)$ associated to $T$ is not completely continuous, because

$$
\left\|T_{1}\left(x_{2}^{n}\right)\right\| \geq\|q\| \cdot\left\|T_{1}\left(x_{2}^{n}\right)\left(x_{1}^{n}, x_{3}^{0}, \ldots, x_{k}^{0}\right)\right\|=\|q\| .
$$

So, $T_{1}$ is not weakly compact, i.e., $T$ is not regular.

Finally, we consider the remaining possibility:

TheOREM 3.4. Let $K_{1}, \ldots, K_{k}$ be infinite compact Hausdorff spaces. Then the following assertions are equivalent:

(a) At least two of the spaces $K_{1}, \ldots, K_{k}$ are not scattered.

$\left(\mathrm{b}_{3}\right) C\left(K_{1}\right) \hat{\otimes}_{\pi} \ldots \hat{\otimes}_{\pi} C\left(K_{k}\right)$ does not have any of the properties DP, $R D P$ and $(\mathrm{V})$.

( $\left.\mathrm{c}_{3}\right) C\left(K_{1}\right) \hat{\otimes}_{\pi} \ldots \hat{\otimes}_{\pi} C\left(K_{k}\right)$ contains a complemented copy of $\ell_{1}$.

$\left(\mathrm{d}_{3}\right)$ There exists a Banach space $X$ and an unconditionally converging multilinear operator $T: C\left(K_{1}\right) \times \ldots \times C\left(K_{k}\right) \rightarrow X$ which is not weakly compact.

Proof. We just show that $\left(\mathrm{a}_{3}\right)$ implies all the others. [13, Proposition 13] states that, if $E \supset \ell_{1}$, then the space $\mathcal{P}\left({ }^{2} E\right)$ of 2-homogeneous polynomials contains a copy of $\ell_{\infty}$. That proof can be easily modified to show that, if both $E$ and $F$ contain copies of $\ell_{1}$, then $\mathcal{L}^{2}(E, F) \supset \ell_{\infty}$. These facts imply that, in that case, $E \hat{\otimes}_{\pi, s} E$ and $E \hat{\otimes}_{\pi} F$ contain complemented copies of $\ell_{1}$. To finish the proof of $\left(\mathrm{c}_{3}\right)$ we just need to observe that $C\left(K_{i}\right) \hat{\otimes}_{\pi} C\left(K_{j}\right)$ is complemented in $C\left(K_{1}\right) \hat{\otimes}_{\pi} \ldots \hat{\otimes}_{\pi} C\left(K_{k}\right)$.

Suppose that $\left(c_{3}\right)$ (and $\left.\left(a_{3}\right)\right)$ hold. Since $\ell_{1}$ is Schur and not reflexive, the projection $\pi: C\left(K_{1}\right) \hat{\otimes}_{\pi} \ldots \hat{\otimes}_{\pi} C\left(K_{k}\right) \rightarrow \ell_{1}$ is an example of a completely continuous (hence unconditionally converging) operator which is not weakly compact. So, $C\left(K_{1}\right) \hat{\otimes}_{\pi} \ldots \hat{\otimes}_{\pi} C\left(K_{k}\right)$ has neither property $(\mathrm{V})$ nor RDPP. The statement about the DP property can be found in [6].

The multilinear operator $\widetilde{\pi}: C\left(K_{1}\right) \times \ldots \times C\left(K_{k}\right) \rightarrow \ell_{1}$ associated to $\pi$ above proves $\left(\mathrm{d}_{3}\right)$.

COROLlary 3.5. Let $K$ be an infinite compact Hausdorff space and $2 \leq$ $k \in \mathbb{N}$. Then the following assertions are equivalent:

(a) $K$ is not scattered.

(b) $\hat{\otimes}_{\pi, s}^{k} C(K)$ does not have properties $D P, R D P P$ and $(\mathrm{V})$.

(c) $\hat{\otimes}_{\pi, s}^{k} C(K)$ contains a complemented copy of $\ell_{1}$.

(d) There exists a $k$-homogeneous unconditionally converging polynomial on $C(K)$ which is not weakly compact.

Proof. As we already said in Corollary 3.2, we only have to show that (a) implies all the others. As above, (c) follows from [13, Proposition 13] and the fact that $\hat{\otimes}_{\pi, s}^{2} C(K)$ is complemented in $\hat{\otimes}_{\pi, s}^{k} C(K)$. 
Let us prove (b): The statement about the DP property can again be found in [6]. As before, the projection $\pi: \hat{\otimes}_{\pi, s}^{k} C(K) \rightarrow \ell_{1}$ is a completely continuous and unconditionally converging operator which is not weakly compact.

The multilinear symmetric operator $\widetilde{\pi}: C(K) \times \stackrel{(k)}{.} \times C(K) \rightarrow \ell_{1}$ associated to $\pi$ proves $(\mathrm{d})$.

\section{Relationships between some classes of multilinear operators} and their linearizations. Let us start this section with a simple and essentially known result which relates the complete continuity of a multilinear operator $T$ and its linearization $\widehat{T}$ :

Proposition 4.1. Let $E_{1}, \ldots, E_{k}$ be Banach spaces. Then the following assertions are equivalent:

(a) For every Banach space $X$, if $\widehat{T}: E_{1} \hat{\otimes}_{\pi} \ldots \hat{\otimes}_{\pi} E_{k} \rightarrow X$ is completely continuous then the associated multilinear operator $T: E_{1} \times \ldots \times E_{k} \rightarrow X$ is completely continuous.

(b) Every multilinear form $\varphi \in \mathcal{L}^{k}\left(E_{1}, \ldots, E_{k}\right)$ is weakly sequentially continuous.

(c) If $\left(x_{i}^{n}\right)_{n} \subset E_{i}(1 \leq i \leq k)$ is a weakly Cauchy sequence, then $\left(x_{1}^{n} \otimes \ldots \otimes x_{k}^{n}\right)_{n} \subset E_{1} \hat{\otimes}_{\pi} \ldots \hat{\otimes}_{\pi} E_{k}$ is weakly Cauchy.

Moreover, if $k=2$, then the condition

(d) $\mathcal{L}\left(E_{1} ; E_{2}^{*}\right)=\mathcal{L}_{\text {cc }}\left(E_{1} ; E_{2}^{*}\right)$

implies all the others.

Proof. The equivalence of (a), (b) and (c) is easy and can be left to the reader. Under hypothesis (d), if $\left(x_{1}^{n}\right) \subset E_{1}$ is a weakly Cauchy sequence and $\left(x_{2}^{n}\right) \subset E_{2}$ is bounded, then $x_{1}^{n} \otimes x_{2}^{n} \subset E_{1} \hat{\otimes}_{\pi} E_{2}$ is weakly Cauchy, which is (c).

So we see that if $\widehat{T}$ is completely continuous, $T$ does not need to be completely continuous. Conversely if $T$ is completely continuous, $\widehat{T}$ need not be completely continuous, even if $E$ and $F$ have the DP (see [6]).

We now study the relation between $T$ being an unconditionally converging multilinear operator and $\widehat{T}$ being an unconditionally converging linear operator. From the previous section it follows that, for every Banach space $X$ and $k \in \mathbb{N}, T \in \mathcal{L}^{k}\left(c_{0} ; X\right)$ is unconditionally converging if and only if $\widehat{T} \in \mathcal{L}\left(c_{0} \hat{\otimes}_{\pi}{ }^{(k)}{ }^{\circ} \hat{\otimes}_{\pi} c_{0} ; X\right)$ is unconditionally converging (if and only if both are weakly compact).

As a consequence we have 
THEOREM 4.2. Let $E_{1}, \ldots, E_{k}$ and $X$ be Banach spaces. If the operator $\widehat{T}: E_{1} \hat{\otimes}_{\pi} \ldots \hat{\otimes}_{\pi} E_{k} \rightarrow X$ is unconditionally converging, then so is $T: E_{1} \times \ldots \times E_{k} \rightarrow X$.

Proof. If $T$ is not unconditionally converging, then there exist w.u.C. series $\sum_{n} x_{1}^{n} \subset E_{1}, \ldots, \sum_{n} x_{k}^{n} \subset E_{k}$ such that $\left(T\left(\sum_{n=1}^{m} x_{1}^{n}, \ldots, \sum_{n=1}^{m} x_{k}^{n}\right)\right)_{m}$ is not a Cauchy sequence. Let $i_{j}: c_{0} \rightarrow E_{j}$ be defined by $i_{j}\left(e_{n}\right)=x_{j}^{n}$ $(1 \leq j \leq k)$. Then the multilinear operator

$$
V: c_{0} \times \stackrel{(k)}{\cdots} \times c_{0} \rightarrow X
$$

defined by

$$
V\left(y_{1}, \ldots, y_{k}\right)=T\left(i_{1}\left(y_{1}\right), \ldots, i_{k}\left(y_{k}\right)\right)
$$

is not unconditionally converging. Hence, $\widehat{V}$ is not unconditionally converging. On the other hand, it is easy to check that $\widehat{V}=\widehat{T} \circ\left(i_{1} \otimes \ldots \otimes i_{k}\right)$, which is unconditionally converging by hypothesis. This contradiction finishes the proof.

The converse of Theorem 4.2 is not true, as the following example shows.

EXAMPLE 4.3. In [7], the authors provide an example of a Banach space $X$ with the RNP (hence not containing $c_{0}$ ) such that $X \hat{\otimes}_{\pi} X$ contains $c_{0}$. Consider the operator

$$
\gamma: X \times X \rightarrow X \hat{\otimes}_{\pi} X
$$

defined by

$$
\gamma(x, y)=x \otimes y
$$

Since $X \not \supset c_{0}, \gamma$ is unconditionally converging (see [4, Proposition 2.10]), but $\widehat{\gamma}$, which is the identity on $X \hat{\otimes}_{\pi} X$, is not unconditionally converging, since it fixes a copy of $c_{0}$.

We have not been able to find a less exotic example. Observe that one can prove that every bilinear operator $T: \ell_{\infty} \times \ell_{\infty} \rightarrow c_{0}$ is unconditionally converging, so any non-unconditionally converging operator (for example any projection) $P: \ell_{\infty} \hat{\otimes}_{\pi} \ell_{\infty} \rightarrow c_{0}$ would provide a more familiar counterexample. We have not been able to find such an object, in particular we do not know if $\ell_{\infty} \hat{\otimes}_{\pi} \ell_{\infty}$ contains complemented copies of $c_{0}$.

From Theorem 4.2 it follows easily that if every unconditionally converging bilinear operator on $E \times F$ is weakly compact, then $E \hat{\otimes}_{\pi} F$ has property $(\mathrm{V})$. We do not believe the converse to be true, but we do not have a counterexample. For a wide variety of spaces the converse is true. For $C(K)$ spaces, this follows from Section 3, but more generally we have

Proposition 4.4. Let $E$ and $F$ be Banach spaces such that $E \hat{\otimes}_{\pi} F$ has property (V). Assume further that at least one of the following conditions holds: 
(1) $E^{*}$ or $F^{*}$ has the metric approximation property.

(2) E or $F$ has an unconditional compact expansion of the identity.

(3) $E^{*}$ or $F^{*}$ has the compact approximation property and is a subspace of a Banach space $Z$ possessing an unconditional compact expansion of the identity.

Then every unconditionally converging bilinear operator on $E \times F$ is weakly compact.

Proof. Clearly, both $E$ and $F$ have property (V). Moreover, [11, Theorem 7] states that, under the hypothesis, $\mathcal{L}\left(E ; F^{*}\right)=\mathcal{K}\left(E ; F^{*}\right)\left(\right.$ or $\mathcal{L}\left(F ; E^{*}\right)$ $\left.=\mathcal{K}\left(F ; E^{*}\right)\right)$. Now, Proposition 2.12 applies.

For $\ell_{p}$ spaces we can make this last result a little more precise:

Proposition 4.5. Let $1<p_{i}<\infty$. Then the following are equivalent:

(a) $\ell_{p_{1}} \hat{\otimes}_{\pi} \ldots \hat{\otimes}_{\pi} \ell_{p_{k}}$ has property $(\mathrm{V})$.

(b) $\mathcal{L}\left(\ell_{p_{1}} \hat{\otimes}_{\pi} \ldots \hat{\otimes}_{\pi} \ell_{p_{k-1}} ; \ell_{q_{k}}\right)=\mathcal{K}\left(\ell_{p_{1}} \hat{\otimes}_{\pi} \ldots \hat{\otimes}_{\pi} \ell_{p_{k-1}} ; \ell_{q_{k}}\right)$, where $\ell_{p_{k}}^{*}=\ell_{q_{k}}$.

(c) $\ell_{p_{1}} \hat{\otimes}_{\pi} \ldots \hat{\otimes}_{\pi} \ell_{p_{k}}$ is reflexive.

(d) $\sum_{i=1}^{k} 1 / p_{i}<1$.

(e) Every unconditionally converging multilinear operator on $\ell_{p_{1}} \times \ldots \times$ $\ell_{p_{k}}$ is weakly compact.

Proof. If (a) holds, then (b) follows from [11, Theorem 7]. The equivalence of (b), (c) and (d) can be found in [1, Section 4].

Observe that, in general, if $E_{1}, \ldots, E_{k}$ are reflexive spaces, then $E_{1} \hat{\otimes}_{\pi}$ $\ldots \hat{\otimes}_{\pi} E_{k}$ is reflexive if and only if every unconditionally converging multilinear operator defined on $E_{1} \times \ldots \times E_{k}$ is weakly compact. For the non-trivial implication of this statement, it suffices to realize that the multilinear operator

$$
T: E_{1} \times \ldots \times E_{k} \rightarrow E_{1} \hat{\otimes}_{\pi} \ldots \hat{\otimes}_{\pi} E_{k}
$$

given by

$$
T\left(x_{1}, \ldots x_{k}\right)=x_{1} \otimes \ldots \otimes x_{k}
$$

is unconditionally converging (this can be seen, for instance, by applying [4, Proposition 2.10] as in Example 4.3). Therefore, (c) and (e) are equivalent. We already mentioned that (e) implies (a) always, i.e., not only for $\ell_{p}$ spaces.

\section{References}

[1] R. Alencar and K. Floret, Weak-strong continuity of multilinear mappings and the Pełczyński-Pitt theorem, J. Math. Anal. Appl. 206 (1997), 532-546.

[2] R. M. Aron, C. Hervés and M. Valdivia, Weakly continuous mappings on Banach spaces, J. Funct. Anal. 52 (1983), 189-204. 
[3] F. Bombal, Sobre algunas propiedades de espacios de Banach, Rev. Acad. Cienc. Madrid 84 (1990), 83-116.

[4] F. Bombal, M. Fernández and I. Villanueva, Unconditionally converging multilinear operators, Math. Nachr. 226 (2001), 5-15.

[5] F. Bombal and I. Villanueva, Regular multilinear operators on $C(K)$ spaces, Bull. Austral. Math. Soc. 60 (1999), 11-20.

[6] - - - On the Dunford-Pettis property of the tensor product of $C(K)$ spaces, Proc. Amer. Math. Soc. 129 (2001), 1359-1363.

[7] J. Bourgain and G. Pisier, A construction of $\mathcal{L}_{\infty}$-spaces and related Banach spaces, Bol. Soc. Brasil. Mat. 14 (1983), 109-123.

[8] J. Diestel, Sequences and Series in Banach Spaces, Grad. Texts in Math. 92, Springer, Berlin 1984.

[9] J. Diestel, H. Jarchow and A. Tonge, Absolutely Summing Operators, Cambridge Stud. in Adv. Math. 43, Cambridge Univ. Press, Cambridge, 1995.

[10] G. Emmanuele, On the reciprocal Dunford-Pettis property in projective tensor products, Math. Proc. Cambridge Philos. Soc. 109 (1991), 161-166.

[11] G. Emmanuele and W. Hensgen, Property (V) of Petczyński in projective tensor products, Proc. Roy. Irish Acad. Sect. A 95 (1995), 227-231.

[12] M. Fernández Unzueta, Unconditionally convergent polynomials in Banach spaces and related properties, Extracta Math. 12 (1997), 305-307.

[13] M. González and J. Gutiérrez, Unconditionally converging polynomials on Banach spaces, Math. Proc. Cambridge Philos. Soc. 117 (1995), 321-331.

[14] A. Grothendieck, Sur les applications linéaires faiblement compactes d'espaces du type $C(K)$, Canad. J. Math. 5 (1953), 129-173.

[15] J. Gutiérrez and I. Villanueva, Extensions of multilinear operators and Banach space properties, preprint.

[16] T. Leavelle, The reciprocal Dunford-Pettis property, preprint.

[17] J. Mujica, Complex Analysis in Banach Spaces, North-Holland Math. Stud. 120, North-Holland, Amsterdam 1986.

[18] A. Pełczyński, Banach spaces on which every unconditionally converging operator is weakly compact, Bull. Acad. Polon. Sci. Sér. Sci. Math. Astronom. Phys. 10 (1962), 641-648.

[19] A. Pełczyński and Z. Semadeni, Spaces of continuous functions (III). Spaces $C(\Omega)$ for $\Omega$ without perfect subsets, Studia Math. 18 (1959), 211-222.

Departamento de Análisis Matemático

CIMAT, A.P. 402

Facultad de Matemáticas

Universidad Complutense de Madrid

Guanajuato, Gto. 36000, México

Madrid 28040, Spain

E-mail: bombal@eucmax.sim.ucm.es ignacio_villanueva@mat.ucm.es

Received September 11, 2000

Revised version September 7, 2001 